\title{
ESTRUTURA DO COMPONENTE ARBÓREO DAS FLORESTAS CILIARES NA BACIA Hidrográfica do Rio Urussanga, sul de Santa Catarina, Brasil
}

\author{
Humberto de Bona Martins", Gullherme Alves Elias ${ }^{1}$, Karoline Ceron², Altamir Rocha Antunes, \\ Renato Colares ${ }^{1}$, Robson dos Santos ${ }^{1}$, Vanilde Citadini-Zanette ${ }^{1}$
}

1 Universidade do Extremo Sul Catarinense (UNESC), Herbário Pe. Dr. Raulino Reitz. (CRI). Av. Universitária, 1105, Bairro Universitário, 88806-000 Criciuma, Santa Catarina, Brasil.

2 Universidade Federal de Mato Grosso do Sul (UFMS), Laboratório de Sistemática e Biogeografia de Anfibios e Répteis, Instituto de Biociências, Cidade Universitária, CEP 79002-970, Campo Grande, Mato Grosso do Sul, Brasil

Recebido em 29 de janeiro de 2018. Aceito em 14 de dezembro de 2019. Publicado em 27 de dezembro de 2019.

Resumo - As florestas ciliares no sul de Santa Catarina têm sofrido recorrentes impactos, principalmente pela introdução de espécies animais e vegetais exóticas, pela pressão agrícola, pelo crescimento das cidades e pela extração do carvão mineral, sendo, no entanto, estruturalmente pouco conhecidas. Este estudo teve como objetivo determinar a estrutura do componente arbóreo em seus distintos estágios sucessionais em 29 fragmentos de Floresta Pluvial subtropical na bacia hidrográfica do rio Urussanga. Em cada fragmento florestal foram alocadas duas parcelas de $10 \mathrm{~m} \times 10 \mathrm{~m}$, perpendiculares à margem do rio, onde foram amostrados os indivíduos arbóreos com diâmetro do caule à altura do peito (DAP) $\geq 5 \mathrm{~cm}$. No total foram amostradas 119 espécies arbóreas. Os fragmentos em estágio médio de sucessão apresentaram maior riqueza $(\mathrm{n}=86)$, seguido pelos de estágio avançado $(n=73)$ e em estágio inicial $(n=59)$, evidenciando que os fragmentos florestais ao longo da bacia hidrográfica do rio Urussanga se encontram em pleno processo de restauração ambiental.

Palavras-chave: mata atlântica, vegetação ripária, biodiversidade, sucessão ecológica secundária, sul do Brasil.

Structure of the arboreal component of Ciliary forests in Rio Urussanga River basin, southern of Santa Catarina, Brazil

Aвstract - Riparian forests in southern Santa Catarina have suffered recurring impacts, mainly due to the introduction of exotic animal and plant species, agricultural pressure, the growth of cities and the extraction of coal, but are structurally little known. This study aimed to determine the structure of the tree component in its distinct successional stages in 29 fragments of subtropical rainforest in the Urussanga river basin. In each forest fragment were allocated two $10 \mathrm{~m} \times 10 \mathrm{~m}$ plots, perpendicular to the river bank, where we sampled tree individuals with stem diameter at breast height (DBH) $\geq 5 \mathrm{~cm}$. A total of 119 tree species were sampled. The medium succession fragments presented higher richness $(\mathrm{n}=86)$, followed by the advanced stage $(n=73)$ and early stage $(n=59)$, showing that the forest fragments along the Urussanga river basin are in the process of environmental restoration.

KEYwORDs: riparian vegetation, biodiversity, secondary ecological succession, southern Brazil.

Estructura del COMPONENTE ARBóREO DE LOS BOSQUES DE RIBERA EN LA CUENCA DEL Río URUSSANGA, AL SUR DE SANTA Catarina, Brasil

Resumen - Los bosques de ribera en el sur de Santa Catarina han sufrido recurrentes impactos, principalmente por la introducción de especies animales y vegetales exóticos, por la presión agrícola, por el crecimiento de las ciudades y la extracción del carbón mineral, siendo, sin embargo, estructuralmente poco conocidos. El objetivo de este estudio fue determinar la 
estructura del componente arbóreo en sus diferentes estadios sucesionales en 29 fragmentos de bosque subtropical en la cuenca del río Urussanga. En cada fragmento forestal se asignaron dos parcelas de $10 \mathrm{~m} \times 10 \mathrm{~m}$, perpendiculares a la orilla del río, donde fueron muestreados los individuos arbóreos con diámetro del tallo a la altura del pecho (DAP) $\geq 5 \mathrm{~cm}$. En total se muestrearon 119 especies arbóreas. Los fragmentos en estadio medio de sucesión presentaron mayor riqueza $(\mathrm{n}=$ 86), seguido por los de estadio avanzado $(n=73)$ y en estadio inicial $(n=59)$, evidenciando que los fragmentos forestales a lo largo de la cuenca del río Urussanga se encuentran en pleno proceso de restauración ambiental.

Palabras clave: bosque atlántico, vegetación riparia, biodiversidad, sucesión ecológica secundaria, sur de Brasil.

\section{INTRODUÇÃO}

As matas ciliares ou matas de galeria são formações florestais presentes ao longo de cursos d'água, desempenhando importantes serviços ecológicos relacionados à qualidade hídrica, além de servir como filtro e retentor de poluentes (Brackmann and Freitas 2013; Jia et al. 2017; Orlowski et al. 2016). Elas proporcionam estabilidade ao relevo e contribuem para a manutenção da biota local, atuando como importantes corredores biológicos (Fernández et al. 2014; Liu et al. 2018; Sun et al. 2018), tais fatores caracterizam esse tipo de formação florestal, como áreas de preservação permanente, protegida por legislação (Rodrigues et al. 2010) no entanto, não impedindo que, no sul de Santa Catarina, ocorressem recorrentes impactos, principalmente pela introdução de espécies exóticas, pela pressão agrícola, pelo crescimento das cidades e, principalmente, pela extração do carvão mineral (Santos et al. 2008; Klein et al. 2009).

A degradação das matas ciliares combinada à escassez de estudos, impossibilita a compreensão sobre a dinâmica florestal dessas áreas (Budke et al. 2004). Em Santa Catarina poucos estudos foram realizados nestas formações (Citadini-Zanette et al. 2014; Colonetti et al. 2009; Reitz and Klein 1964; Sevegnani et al. 2012). Especificamente, na bacia hidrográfica do Rio Urussanga, sul do estado de Santa Catarina, não há estudos em remanescentes de matas ciliares, embora a região esteja inserida como a $14^{a}$ área ambientalmente crítica do Brasil, devido à intensa atividade de lavra e beneficiamento do carvão mineral (Ravazzoli 2013).

Comumente, os estudos desenvolvidos em matas ciliares, restringem a descrição das florestas a uma visão horizontal, negligenciando detalhes importantes da composição florístico-estrutural (Rodrigues et al. 2010). Portanto, há necessidade de um aprimoramento das descrições, no que diz respeito à fisionomia das matas ciliares, de maneira que permita correlações mais estreitas da vegetação (Santos et al. 2016), uma vez que esses ambientes são diversos e podem apresentar todos os tipos de estrutura e estágio sucessional (Fierke and Kauffman 2005). Neste contexto, a influência hidrográfica e edáfica são determinantes no estabelecimento das espécies nesses locais, proporcionando à floresta composição e estrutura peculiares (Rodrigues e Shepherd 1992).

Diante do exposto, este estudo teve por objetivo caracterizar o componente arbóreo das matas ciliares na bacia hidrográfica do Rio Urussanga, sul do estado de Santa Catarina.

\section{Material e MÉtodos}

A amostragem foi realizada no período de novembro de 2012 a maio de 2014, em fragmentos de Floresta Pluvial subtropical (Oliveira-Filho 2009) na Bacia Hidrográfica do Rio Urussanga, município de Urussanga, Santa Catarina, Brasil (Figura 1). No município predominam Argissolos e Cambissolos (Back 2009). O clima é classificado, segundo a classificação de Köppen, como Cfa, sem estação seca definida e com verões quentes (Alvares et al. 2013), com índices pluviométricos médios de $1.400 \mathrm{~mm}$ ao ano e umidade relativa do ar de 82\% (Back 2009). 
Figura 1. Localização da área de estudo e dos locais de amostragem para o levantamento do componente arbóreo ciliar na Bacia Hidrográfica do Rio Urussanga, município de Urussanga, sul de Santa Catarina, Brasil.

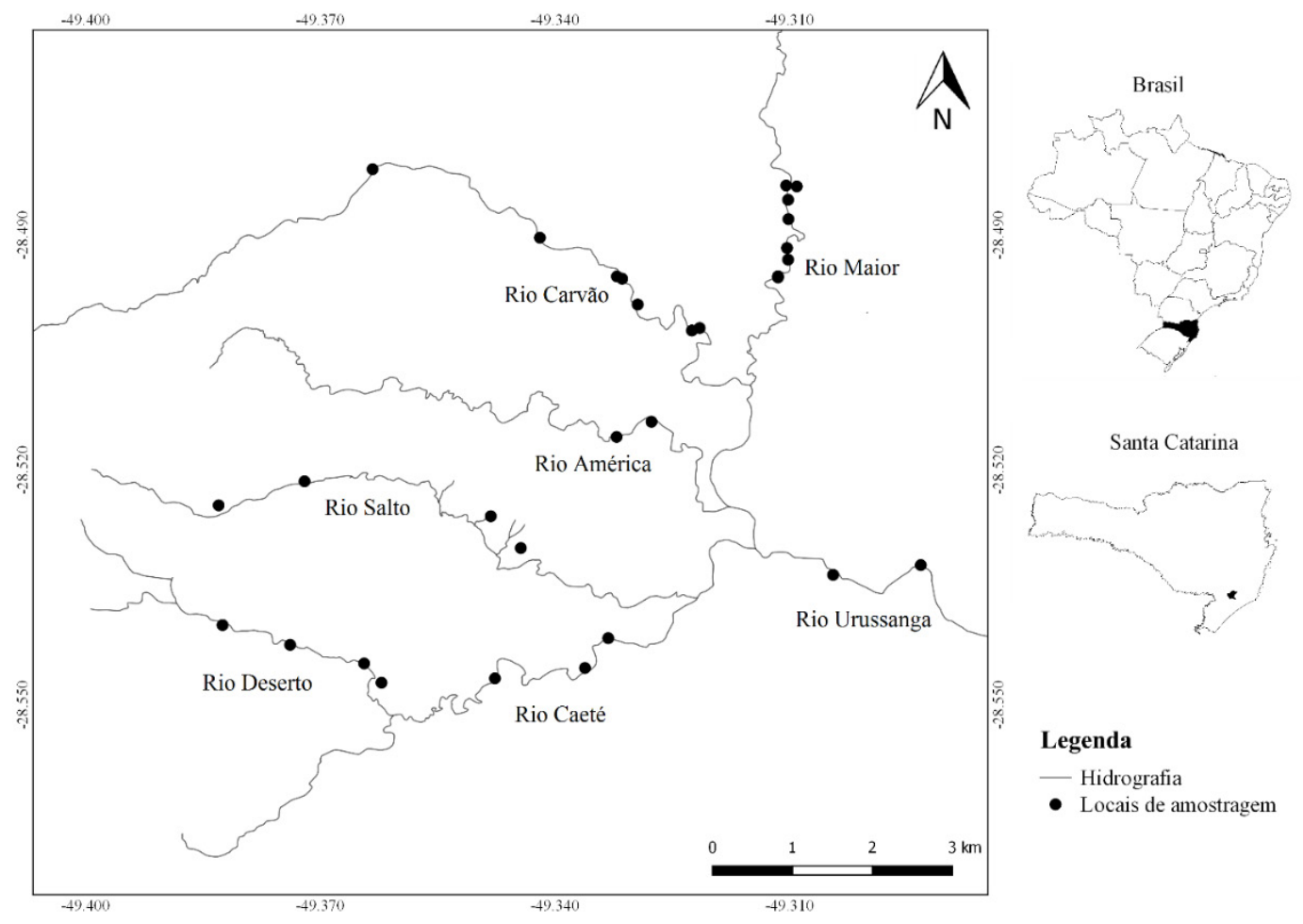

O levantamento do componente arbóreo ciliar foi realizado em 29 fragmentos florestais, localizados em sete rios (América, Caeté, Carvão, Deserto, Maior, Salto e Urussanga) pertencentes à Bacia Hidrográfica do Rio Urussanga (Figura 1). Em cada fragmento florestal foram alocadas duas parcelas de $10 \mathrm{~m} \times 10 \mathrm{~m}$, perpendiculares à margem do rio, com distância de $1 \mathrm{~m}$. Foram amostrados todos os indivíduos arbóreos, incluindo palmeiras e samambaias com hábito arborescente, que apresentaram Diâmetro à Altura do Peito (DAP) $\geq 5 \mathrm{~cm}$, índice sugerido para amostragem do componente arbóreo-arbustivo no domínio Mata Atlântica (Moro e Martins 2011).

Para classificação dos fragmentos florestais de acordo com seu estágio sucessional foi considerado a soma da densidade relativa das espécies de início de sucessão (pioneiras + secundárias iniciais). Desse modo, locais com densidade das espécies de início de sucessão superior a $60 \%$, o fragmento florestal foi considerado em estágio inicial, entre 30\% e 60\%, em estágio médio e, inferiores a 30\%, em estágio avançado de regeneração natural. Posteriormente, procedeu-se o cálculo dos parâmetros fitossociológicos de frequência, densidade e dominância, além do valor de importância (Mueller-Dombois and Ellenberg 2002). As espécies foram classificadas conforme os grupos ecológicos (Budowski 1965).

Para avaliar a diferença nos atributos de riqueza e abundância por parcela e entre os diferentes estádios sucessionais propostos, foi realizado o teste de Kruskull-Wallis (Sijtsma and Emons 2010). Este teste permite a comparação sem necessitar que os grupos tenham o mesmo tamanho amostral.

A atualização taxonômica seguiu a Lista das Espécies da Flora do Brasil (FLORA DO BRASIL 2020, EM CONSTRUÇÃO) e a delimitação das famílias botânicas das espécies encontradas seguiu o APG IV (2016) para as angiospermas e Smith et al. (2006) para as samambaias.

\section{REsultados}

Os fragmentos florestais foram agrupados em seus respectivos estágios sucessionais: sete em estágio inicial, 13 em estágio médio e nove em estágio avançado de regeneração natural. Somando-se a fitodiversidade desses 
fragmentos florestais nos três estágios sucessionais foram amostradas 119 espécies (Tabelas 1 a 3). Por uma maior clareza e refinamento dos dados, nas tabelas 1, 2 e 3, foram priorizadas as espécies com maior valor importância (VI).

No estágio inicial de sucessão 20 espécies foram classificadas como pioneiras, 14 como secundárias iniciais, 17 como secundárias tardias e oito como espécies clímax. Dentre estas, as que obtiveram maior Valor de Importância (VI) foram A. triplinervia, $M$. splendens e $P$. gonoacantha.

Tabela 1. Espécies arbóreas presentes na floresta ciliar em estágio inicial de sucessão na Bacia Hidrográfica do Rio Urussanga, Sul de Santa Catarina, e seus respectivos descritores estruturais, em que: FA= Frequência

Absoluta (\%), FR= Frequência Relativa (\%), DA= Densidade Absoluta (indivíduos.ha ${ }^{-1}$ ), DR= Densidade Relativa (\%), DoA= Dominância Absoluta $\left(\mathrm{m}^{2} \cdot \mathrm{ha}^{-1}\right), \mathrm{DoR}=$ Dominância Relativa e VI= Valor de Importância; Grupo Ecológico (GE), onde: Pio $=$ Pioneira, $\operatorname{Sin}=$ Secundária inicial, Sta $=$ Secundária Tardia e Cli $=$ Clímax

\begin{tabular}{|c|c|c|c|c|c|c|c|c|c|}
\hline Espécies & Nome Popular & FA & FR & DA & DR & DoA & DoR & VI & GE \\
\hline Alchornea triplinervia (Spreng.) Müll.Arg. & tanheiro & 57,1 & 7,0 & 292,9 & 18,1 & 6,83 & 27,63 & 52,7 & Sin \\
\hline Myrcia splendens (Sw.) DC. & guamirim-de-folha-fina & 64,3 & 7,9 & 157,1 & 9,7 & 1,9 & 7,69 & 25,3 & Sta \\
\hline Piptadenia gonoacantha (Mart.) J.F.Macbr. & pau-jacaré & 21,4 & 2,6 & 50,0 & 3,1 & 2,68 & 10,84 & 16,6 & Pio \\
\hline Nectandra membranacea (Sw.) Griseb. & canela-caqui-branca & 21,4 & 2,6 & 42,9 & 2,6 & 1,33 & 5,38 & 10,7 & Sta \\
\hline Tetrorchidium rubrivenium Poepp. \& Endl. & embirão & 35,7 & 4,4 & 35,7 & 2,2 & 0,63 & 2,55 & 9,1 & $\operatorname{Sin}$ \\
\hline Syagrus romanzoffiana (Cham.) Glassman & jerivá & 14,3 & 1,8 & 28,6 & 1,8 & 1,4 & 5,66 & 9,2 & Sta \\
\hline Matayba intermedia Radlk. & camboatá-branco & 28,6 & 3,5 & 35,7 & 2,2 & 0,79 & 3,20 & 8,9 & Sta \\
\hline Tabernaemontana catharinensis DC. & leiteiro & 14,3 & 1,8 & 57,1 & 3,5 & 0,66 & 2,67 & 7,9 & Pio \\
\hline Allophylus edulis (A.St.-Hil. et al.) Hieron. ex Niederl. & chal-chal & 21,4 & 2,6 & 50,0 & 3,1 & 0,51 & 2,06 & 7,8 & $\operatorname{Sin}$ \\
\hline Cabralea canjerana (Vell.) Mart. & cabralea & 28,6 & 3,5 & 42,9 & 2,6 & 0,36 & 1,46 & 7,6 & Sta \\
\hline Miconia cabucu Hoehne & pixiricão & 14,3 & 1,8 & 71,4 & 4,4 & 0,35 & 1,42 & 7,6 & Pio \\
\hline Myrcia brasiliensis Kiaersk. & grumixama & 21,4 & 2,6 & 28,6 & 1,8 & 0,79 & 3,20 & 7,6 & Sta \\
\hline Jacaranda puberula Cham. & carobinha & 28,6 & 3,5 & 35,7 & 2,2 & 0,18 & 0,73 & 6,4 & Pio \\
\hline Psychotria vellosiana Benth. & café-do-mato & 28,6 & 3,5 & 28,6 & 1,8 & 0,25 & 1,01 & 6,3 & Sta \\
\hline Esenbeckia grandiflora Mart. & cutia & 7,1 & 0,9 & 64,3 & 4,0 & 0,31 & 1,25 & 6,1 & $\operatorname{Sin}$ \\
\hline Casearia obliqua Spreng. & guaçatonga & 14,3 & 1,8 & 42,9 & 2,6 & 0,37 & 1,50 & 5,9 & $\operatorname{Sin}$ \\
\hline Myrsine coriacea (Sw.) R. Br & capororoca & 14,3 & 1,8 & 35,7 & 2,2 & 0,29 & 1,17 & 5,1 & $\operatorname{Sin}$ \\
\hline Trema micrantha (L.) Blume & grandiúva & 14,3 & 1,8 & 35,7 & 2,2 & 0,24 & 0,97 & 4,9 & Pio \\
\hline Euterpe edulis Mart. & palmiteiro & 21,4 & 2,6 & 28,6 & 1,8 & 0,12 & 0,49 & 4,9 & Cli \\
\hline Nectandra oppositifolia Nees & canela-ferrugem & 14,3 & 1,8 & 21,4 & 1,3 & 0,27 & 1,09 & 4,2 & Sta \\
\hline Outras espécies* & - & 327,3 & 41,4 & 434,8 & 26,1 & 4,46 & 17,99 & 85,5 & - \\
\hline TOTAIS & & 814,3 & 100 & 1621,4 & 100 & 24,72 & 100 & 300 & \\
\hline
\end{tabular}

No estágio médio de sucessão 21 espécies foram classificadas como pioneiras, 26 como secundárias iniciais, 22 como secundárias tardias e 17 como clímax. As espécies com maior VI foram $H$. alchorneoides, Euterpe edulis e M. intermedia.

Tabela 2. Espécies arbóreas presentes na floresta ciliar em estágio médio de sucessão na Bacia Hidrográfica do Rio Urussanga, Sul de Santa Catarina, e seus respectivos descritores estruturais, em que: FA= Frequência Absoluta (\%), FR= Frequência Relativa (\%), DA= Densidade Absoluta (indivíduos.ha ${ }^{-1}$ ), DR= Densidade Relativa (\%), DoA= Dominância Absoluta $\left(\mathrm{m}^{2} \cdot \mathrm{ha}^{-1}\right), \mathrm{DoR}=$ Dominância Relativa e VI= Valor de Importância; Grupo Ecológico (GE), onde: Pio= Pioneira, $\operatorname{Sin}=$ Secundária inicial, Sta $=$ Secundária Tardia e Cli $=$ Clímax .

\begin{tabular}{|c|c|c|c|c|c|c|c|c|c|}
\hline Espécies & Nome Popular & FA & FR & DA & DR & DoA & DoR & VI & GE \\
\hline Hieronyma alchorneoides Allemão & licurana & 14,3 & 4,0 & 84,6 & 3,7 & 4,2 & 13,06 & 20,8 & $\operatorname{Sin}$ \\
\hline Euterpe edulis Mart. & palmiteiro & 17,5 & 4,9 & 207,7 & 9,1 & 1,35 & 4,20 & 18,2 & Cli \\
\hline Matayba intermedia Radlk. & camboatá-branco & 15,9 & 4,5 & 161,5 & 7,1 & 2,05 & 6,37 & 17,9 & Sta \\
\hline Nectandra oppositifolia Nees & canela-ferrugem & 14,3 & 4,0 & 84,6 & 3,7 & 2,07 & 6,43 & 14,2 & Sta \\
\hline
\end{tabular}




\begin{tabular}{|c|c|c|c|c|c|c|c|c|c|}
\hline Espécies & Nome Popular & FA & FR & DA & DR & DoA & DoR & VI & GE \\
\hline Alsophila setosa Kaulf. & samambaiaçu & 4,8 & 1,3 & 180,8 & 7,9 & 1,18 & 3,67 & 12,9 & Cli \\
\hline Cabralea canjerana (Vell.) Mart. & cabralea & 14,3 & 4,0 & 96,2 & 4,2 & 1,18 & 3,67 & 11,9 & Sta \\
\hline Lamanonia ternata Vell. & sacopema & 6,4 & 1,8 & 73,1 & 3,2 & 1,6 & 4,97 & 10,0 & Pio \\
\hline Clethra scabra Pers. & carne-de-vaca & 6,4 & 1,8 & 65,4 & 2,9 & 1,57 & 4,88 & 9,5 & Pio \\
\hline Casearia sylvestris Sw. & cháde-bugre & 11,1 & 3,1 & 53,9 & 2,4 & 1,18 & 3,67 & 9,1 & Sin \\
\hline Alchornea triplinervia (Spreng.) Müll.Arg. & tanheiro & 7,9 & 2,2 & 65,4 & 2,9 & 1,27 & 3,95 & 9,0 & $\operatorname{Sin}$ \\
\hline Myrcia splendens (Sw.) DC. & guamirim-da-folha-fina & 7,9 & 2,2 & 96,2 & 4,2 & 0,77 & 2,39 & 8,8 & $\operatorname{Sin}$ \\
\hline Miconia cabucu Hoehne & pixiricão & 11,1 & 3,1 & 57,7 & 2,5 & 1,01 & 3,14 & 8,8 & Pio \\
\hline Psychotria vellosiana Benth. & café-do-mato & 9,5 & 2,7 & 73,1 & 3,2 & 0,57 & 1,77 & 7,6 & Sta \\
\hline Piptadenia gonoacantha (Mart.) J.F.Macbr. & pau-jacaré & 7,9 & 2,2 & 42,3 & 1,8 & 1,1 & 3,42 & 7,5 & Pio \\
\hline Syagrus romanzoffiana (Cham.) Glassman & jerivá & 3,2 & 0,9 & 30,8 & 1,3 & 1,22 & 3,79 & 6,0 & Sta \\
\hline Ocotea odorifera (Vell.) Rohwer & sassafrás & 3,2 & 0,9 & 69,2 & 3,0 & 0,61 & 1,90 & 5,8 & Cli \\
\hline Machaerium stipitatum Vogel & farinha-seca & 6,4 & 1,8 & 53,9 & 2,4 & 0,5 & 1,55 & 5,7 & Pio \\
\hline Nectandra megapotamica (Spreng.) Mez & canela-imbuia & 6,4 & 1,8 & 38,5 & 1,7 & 0,65 & 2,02 & 5,5 & Cli \\
\hline Outras espécies* & - & 188,5 & 50,4 & 755,6 & 33,2 & 8,09 & 25,09 & 110,4 & - \\
\hline TOTAIS & & 355,6 & 100 & 2288,5 & 100 & 32,17 & 100 & 300 & \\
\hline
\end{tabular}

No estágio avançado de sucessão sete espécies foram classificadas como pioneiras, 12 como secundárias iniciais, 34 como secundárias tardias e 19 como clímax. As espécies com maior VI foram Euterpe edulis, Cabralea canjerana e Hieronyma alchorneoides.

Tabela 3. Espécies arbóreas presentes na floresta ciliar em estágio avançado de sucessão na Bacia Hidrográfica do Rio Urussanga, Sul de Santa Catarina, e seus respectivos descritores estruturais, em que: FA= Frequência

Absoluta (\%), FR= Frequência Relativa (\%), DA= Densidade Absoluta (indivíduos.ha ${ }^{-1}$ ), $D R=$ Densidade Relativa (\%), DoA= Dominância Absoluta $\left(\mathrm{m}^{2} \cdot \mathrm{ha}^{-1}\right), \mathrm{DoR}=$ Dominância Relativa e VI= Valor de Importância; Grupo Ecológico (GE), onde: Pio= Pioneira, $\operatorname{Sin}=$ Secundária inicial, Sta $=$ Secundária Tardia e Cli $=$ Clímax.

\begin{tabular}{|c|c|c|c|c|c|c|c|c|c|}
\hline Espécies & Nome Popular & FA & FR & DA & DR & DoA & DoR & VI & GE \\
\hline Euterpe edulis Mart. & palmiteiro & 77,8 & 7,9 & 316,7 & 17,3 & 2,47 & 6,79 & 32,0 & Cli \\
\hline Cabralea canjerana (Vell.) Mart. & cabralea & 55,6 & 5,6 & 144,4 & 7,9 & 2,57 & 7,05 & 20,6 & Sta \\
\hline Hieronyma alchorneoides Allemão. & licuranta & 38,9 & 3,9 & 61,1 & 3,3 & 4,69 & 12,86 & 20,1 & $\operatorname{Sin}$ \\
\hline Alchornea triplinervia (Spreng.) Müll.Arg. & tanheiro & 33,3 & 3,4 & 38,9 & 2,1 & 4,44 & 12,19 & 17,7 & $\operatorname{Sin}$ \\
\hline Matayba intermedia Radlk. & camboatá-branco & 33,3 & 3,4 & 94,4 & 5,2 & 2,02 & 5,54 & 14,1 & Sta \\
\hline Cyathea delgadii Sternb. & samambaia & 50,0 & 5,1 & 88,9 & 4,9 & 0,80 & 2,20 & 12,1 & Cli \\
\hline Alsophila setosa Kaulf. & samambaiaçu & 38,9 & 3,9 & 100,0 & 5,5 & 0,83 & 2,27 & 11,7 & Cli \\
\hline Nectandra oppositifolia Nees & canela-ferrugem & 27,8 & 2,8 & 38,9 & 2,1 & 2,40 & 6,60 & 11,5 & Sta \\
\hline Esenbeckia grandiflora Mart. & cutia & 27,8 & 2,8 & 61,1 & 3,3 & 1,06 & 2,90 & 9,1 & $\operatorname{Sin}$ \\
\hline Mollinedia triflora (Spreng.) Tul.. & pimenteira & 27,8 & 2,8 & 55,6 & 3,0 & 0,29 & 0,81 & 6,7 & Cli \\
\hline Mollinedia schottiana (Spreng.) Perkins. & capixim & 27,8 & 2,8 & 50,0 & 2,7 & 0,40 & 1,09 & 6,6 & Cli \\
\hline Guarea macrophylla Vahl & marinheiro & 27,8 & 2,8 & 50,0 & 2,7 & 0,31 & 0,85 & 6,4 & Cli \\
\hline Cedrela fissilis Vell. & cedro & 11,1 & 1,1 & 27,8 & 1,5 & 1,35 & 3,70 & 6,3 & Sta \\
\hline Casearia sylvestris $\mathrm{Sw}$ & chá-de-bugre & 22,2 & 2,2 & 27,8 & 1,5 & 0,82 & 2,24 & 6,0 & $\operatorname{Sin}$ \\
\hline Myrcia splendens (Sw.) DC. & guamirim-de-folha-fina & 16,7 & 1,7 & 33,3 & 1,8 & 0,85 & 2,33 & 5,8 & Sta \\
\hline Tetrorchidium rubrivenium Poepp. \& Endl & embirão & 5,6 & 0,6 & 5,6 & 0,3 & 1,77 & 4,85 & 5,7 & $\operatorname{Sin}$ \\
\hline Ocotea elegans $\mathrm{Mez}$ & canela-parda & 11,1 & 1,1 & 11,1 & 0,6 & 1,38 & 3,78 & 5,5 & Cli \\
\hline Miconia cabucu Hoehne & pixiricão & 22,2 & 2,2 & 27,8 & 1,5 & 0,47 & 1,30 & 5,1 & Pio \\
\hline Outras espécies* & - & 434,9 & 44,9 & 596 & 32,1 & 7,52 & 20,65 & 97,2 & - \\
\hline TOTAIS & & 989,1 & 100 & 1827,9 & 100 & 36,40 & 100 & 300 & \\
\hline
\end{tabular}


Em uma comparação entre os três estágios sucessionais, o estágio médio obteve a maior riqueza de espécies, gêneros e famílias, seguido pelos estágios avançado e inicial (Tabela 4). O índice de similaridade de Jaccard indicou maior similaridade entre o estágio médio e avançado (44\%), seguida do inicial e médio (41\%) e inicial e avançado $(37 \%)$.

A riqueza não diferiu estatisticamente entre os grupos $(\chi: 3,91, \mathrm{p}>0,14)$, sugerindo que a flora arbórea tende a se comportar com riqueza semelhante entre os estádios sucessionais propostos. Para a abundância também não foi encontrado diferença significativa $(\chi: 2,08$, p: 0,35) (Figura 2).

Figura 2. Teste de Kruskull-Wallis representa que os valores de riqueza e abundância são semelhantes em todos os ambientes, portanto não possuem diferenças significativas.

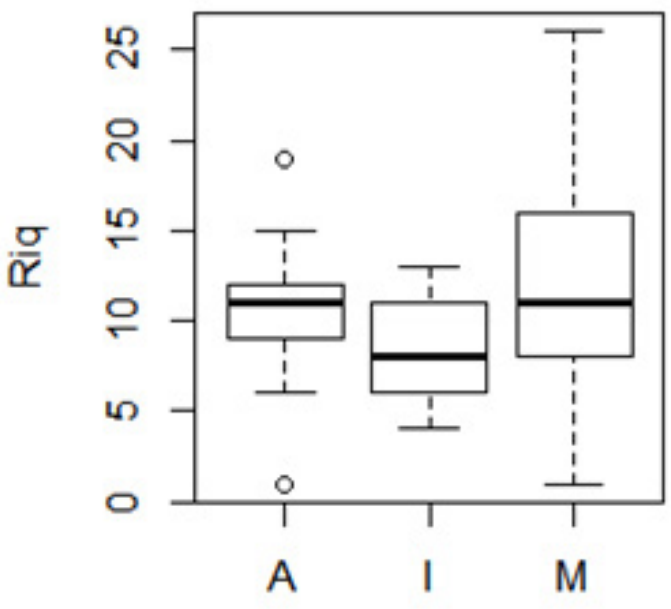

Est

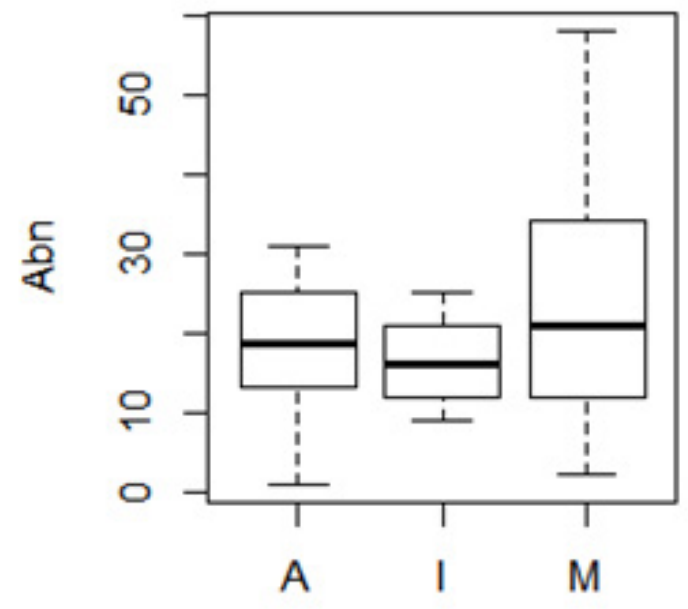

Est

O maior valor para área basal foi no estágio avançado seguido do estágio médio e inicial de regeneração natural. A diversidade $\left(\mathrm{H}^{\prime}\right)$ foi maior no estágio médio e igual para os estágios inicial e avançado de sucessão. A equabilidade (J') foi maior no estágio inicial seguido do estágio médio e avançado, no entanto, com pequena diferença entre eles (Tabela 4).

Tabela 4. Caracterização das fitofisionomias dos estágios sucessionais na floresta ciliar na Bacia Hidrográfica do Rio Urussanga, Sul de Santa Catarina, Brasil. $\mathrm{DA}=$ densidade absoluta e DoA $=$ dominância absoluta $\left(\mathrm{m}^{2} . \mathrm{ha}^{-1}\right) \mathrm{e}$ índice de diversidade (nats/indivíduo).

\begin{tabular}{|c|c|c|c|c|c|c|c|}
\hline \multirow{2}{*}{ Estágio sucessional } & \multirow{2}{*}{ Famílias } & \multirow{2}{*}{ Gêneros } & \multirow{2}{*}{ Espécies } & \multirow{2}{*}{$\mathrm{DA}$} & \multirow{2}{*}{ DoA } & \multicolumn{2}{|c|}{ Índice } \\
\hline & & & & & & Diversidade & Equabilidade \\
\hline Inicial & 26 & 49 & 59 & 1.621 & 24,72 & 3,56 & 0,86 \\
\hline Médio & 39 & 72 & 86 & 2.289 & 32,17 & 3,80 & 0,85 \\
\hline Avançado & 27 & 58 & 73 & 1.828 & 36,40 & 3,56 & 0,83 \\
\hline
\end{tabular}

Percentualmente, o estágio inicial de sucessão apresentou predominância de indivíduos (65\%) de espécies de grupos ecológicos de início de sucessão. No estágio médio o percentual foi de 45\% e no avançado foi de $19 \%$ (Figura 3). Quando avaliado o número de espécies, o percentual obtido para os fragmentos em estágio inicial (58\%) e médio (54\%) ficaram muito próximos, e os fragmentos em estágio avançado obtiveram 25\% (Figura 3). 
Figura 3. Percentual de espécies e indivíduos por grupo ecológico nos três estágios sucessionais (inicial, médio e avançado) amostrados nos trechos de mata ciliar ao longo da Bacia Hidrográfica do Rio Urussanga, sul de Santa Catarina.

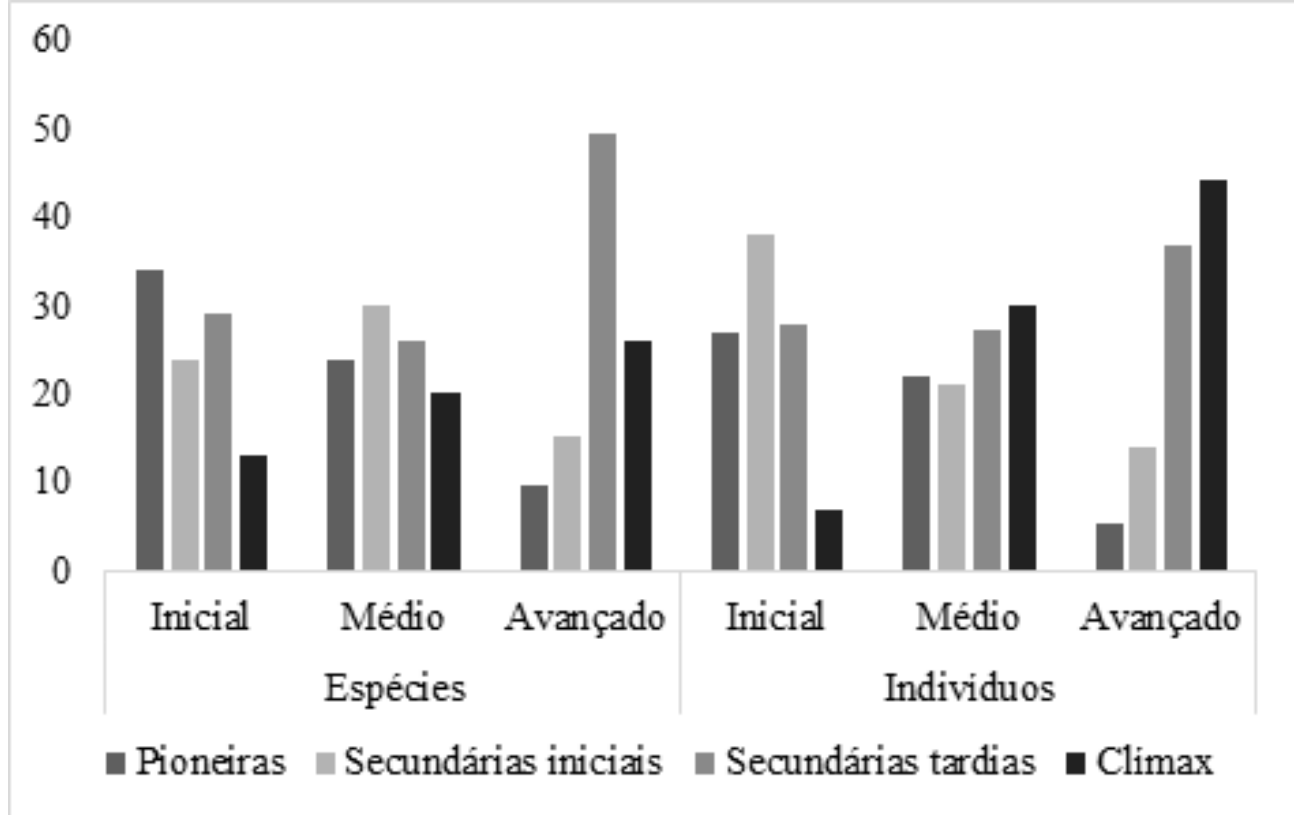

A altura média das espécies amostradas nos estágios inicial, médio e avançado foram de $8,3 \mathrm{~m}, 8,6 \mathrm{~m}$ e $7,7 \mathrm{~m}$, respectivamente (Figura 4).

No estágio inicial as espécies que caracterizam o dossel foram Alchornea triplinervia, Myrcia splendens e Piptadenia gonoacantha, onde foram encontradas algumas espécies emergentes, como Alchornea triplinervia (18 m), Nectandra megapotamica $(17 \mathrm{~m})$ e Tetrorchidium rubrivenium $(17 \mathrm{~m})$. Já o estágio médio e o avançado apresentaram classes de altura similares. As espécies que caracterizaram o dossel dessas florestas foram Euterpe edulis, Hieronyma alchorneoides e Matayba intermedia. Entre as emergentes no estágio médio foram Cupania vernalis (19 m), Piptadenia gonoacantha (18 m) e Hieronyma alchorneoides (18 m) e no avançado foram Nectandra leucantha (18 m) e Alchornea triplinervia (14 m).

A análise de distribuição dos indivíduos amostrados nos três estágios sucessionais em classes diamétricas revelou um padrão em J-invertido, desta maneira apresentando a maior concentração de indivíduos nas classes de menor diâmetro, seguindo um decréscimo em direção às classes maiores (Figura 4). As classes diamétricas entre 5,0-7,9 congregou 41,7\% dos indivíduos no estágio inicial de sucessão. No estágio médio, os indivíduos se distribuiram de maneira gradual entre as duas primeiras classes, sendo 38,82\% entre 5,0-7,9 e 27,39\% entre 8,0$10,9 \mathrm{~cm}$. No estágio avançado, os indivíduos apresentam 38,9\% e 25,83\% nas classes entre 5,0-7,9 e 8,0-10,9 cm, respectivamente.

Os três estágios estudados apresentaram maior número de indivíduos com DAP entre 5-7,9 cm, o que pode estar associado à elevada taxa de regeneração natural presente nos remanescentes estudados (Figura 4). Do mesmo modo, os estágios iniciais, médio e avançado apresentaram predominância de indivíduos com altura entre 6-7,9 m (Figura 4). 
Figura 4. Percentual de indivíduos por estágios sucessional e suas respectivas (a) classes de diâmetro - cm e (b) classes de altura -m, amostrados nos fragmentos de floresta ciliar na Bacia Hidrográfica do Rio Urussanga, sul de Santa Catarina.

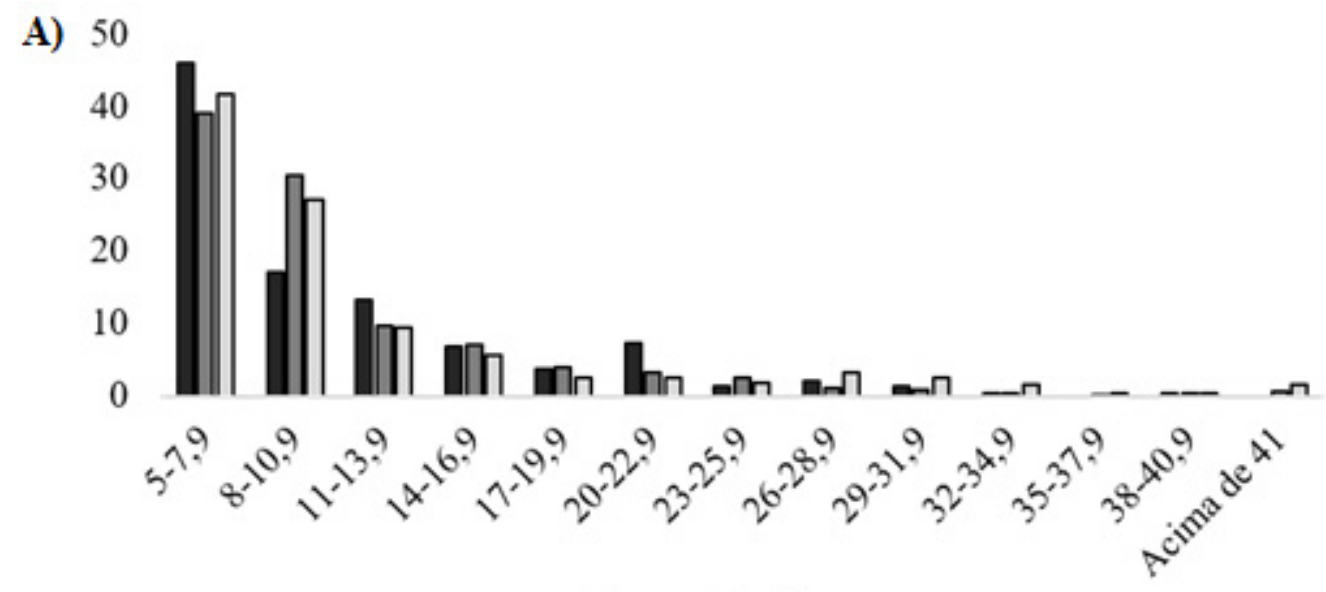

Classes de diâmetro

B) 40

घInicial 口Médio 口Avancado

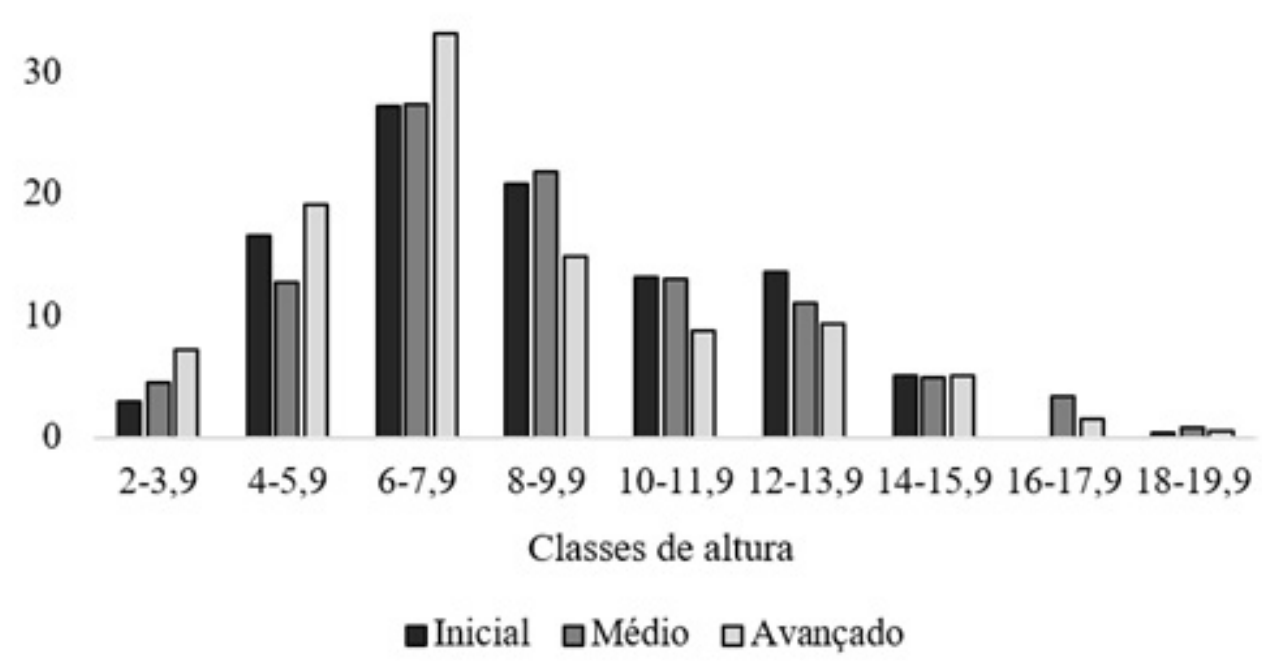

Foram registradas 3 espécies ameaçadas de extinção, sendo elas: Euterpe edulis, Ocotea odorifera e Cedrela fissilis (CNCFLORA, 2017). Em relação a espécies ameaçadas, Euterpe edulis e Ocotea odorifera ocorreram em estágio médio e avançado de regeneração natural enquanto Cedrela fissilis foi registrada em todos os estágios sucessionais.

\section{Discussão}

O estágio sucessional médio apresentou a maior riqueza, se comparado ao estágio inicial e avançado, que pode estar relacionada à posição intermediária do estágio médio, que oferece condições tanto às espécies pioneiras e secundárias iniciais quanto às secundárias tardias e clímax. Esta situação é comum em florestas secundárias, uma vez que são mosaicos de espécies pioneiras, secundárias iniciais e tardias (Leitão Filho e Pagano 1993). Essa evidência corrobora com a teoria do distúrbio intermediário, onde a maior riqueza de espécies encontra-se em níveis intermediários de distúrbio, estando fundamentada na tolerância das espécies ao distúrbio e à habilidade de competição (Connell 1978; Molino e Sabatier 2001). 
A maior riqueza de Lauraceae no estágio inicial e médio é atribuído a sua constante presença nas listas de famílias mais ricas no âmbito da Floresta Atlântica, sobretudo na Floresta Pluvial subtropical (Colonetti et al., 2009). Ainda, a distribuição de Lauraceae no Brasil é ampla, sendo registrada em todo território, com 24 gêneros e 439 espécies (FLORA DO BRASIL 2020, EM CONSTRUÇÃO).

Myrtaceae apresentou alta representatividade neste estudo, apoiando outros estudos desenvolvidos no sul de Santa Catarina (Citadini-Zanette et al. 2014; Colonetti et al. 2009) sendo expressiva no estágio avançado, estando relacionada ao elevado número de espécies com ocorrência natural no estado (cerca de 180 espécies) e no Brasil (cerca de 1.000 espécies), o que a torna uma das maiores famílias da flora brasileira (FLORA DO BRASIL 2020, EM CONSTRUÇÃO). Houve forte incremento na riqueza e abundância de Myrtaceae com o avanço dos estágios sucessionais. No estágio inicial foram registradas cinco espécies, no estágio médio seis e no avançado doze, corroborando estudos que demonstram que em estágios mais conservados de regeneração natural há maior número de espécies de Myrtaceae (Bosa et al. 2015; Colonetti et al. 2009; Leitão Filho e Pagano 1993). Estas espécies possuem alta dependência de interação com a fauna por meio da polinização e da dispersão, o que pode justificar sua grande abundância na Floresta Atlântica, assim como em estágios mais avançados de sucessão. Myrtaceae e Lauraceae são comumente relacionadas em inventários na região sul do Brasil, principalmente nos estágios finais de sucessão, o que denota a importância fitossociológica dessas famílias em ambientes florestais do Brasil (Bosa et al. 2015; Citadini-Zanette et al. 2014; Colonetti et al. 2009; Leitão Filho e Pagano 1993; Reitz e Klein 1964).

A família Euphorbiaceae apresentou os maiores valores de importância nos três estágios sucessionais, podendo ser atribuído à elevada frequência e densidade de suas espécies no levantamento fitossociológico. $A$. triplinervia e $H$. alchorneoides apresentaram os maiores valores de importância no estágio inicial e médio, respectivamente. Estudos próximos à região estudada apontam que $A$. triplinervia ocorre em estágio avançado de regeneração natural, se destacando pela frequência e dominância. Além disso, a espécie ainda apresenta potencial uso econômico, sendo utilizada para reflorestamento, corte da madeira (tábuas) e como medicinal (Trindade e Lameira 2015). Hieronyma alchorneoides é considerada heliófita ou de luz difusa, tornando-se frequente nos capoeirões e nas matas secundárias, formando o início da sucessão para a floresta secundária onde começam a surgir as espécies esciófitas (Smith et al. 1988), fato corroborado por este estudo visto que a espécie teve maior representatividade no estágio médio de sucessão.

No estágio avançado de sucessão, E. edulis apresentou maior valor de importância. Ela é considerada espéciechave dentro dos sistemas florestais por ser abundante e exercer papel fundamental na interação com animais, representando, inclusive, alternativa alimentar em épocas de escassez de alimento na Floresta Atlântica (Reis 1995; Elias et al. 2015; Elias e Santos 2016). É frequente em florestas climácicas associada à Ocotea catharinensis Mez (Reitz e Klein 1974). Euterpe edulis também pode desenvolver-se nos estágios iniciais, como já registrado em capoeiras dominadas por M. coriacea (Reitz e Klein 1974; Ribeiro et al. 2011). No presente estudo foi observado que a espécie inicia sua colonização em ambientes iniciais, com incremento significativo nos ambientes mais conservados, com alta densidade e frequência (Elias e Santos 2016), ratificando os dados da literatura. O fato das espécies apresentarem diferentes exigências e estratégias de ocupação, permite que exista diversidade entre as mais importantes (VI) nos diferentes estágios de sucessão (Lopes et al. 2002).

A predominância de espécies pioneiras e secundárias iniciais no estágio inicial de regeneração, registrada neste estudo, está em consonância com a literatura (Budowski 1965; Peña-Claros 2003; Sawczuk et al. 2012; Venzke e Martins 2013). Leitão Filho (1993) ressalta que, para áreas iniciais, com perturbação ocorrida entre 10 e 15 anos, o percentual de espécies pioneiras e secundárias iniciais chega a 74,2\% com 85,7\% dos indivíduos. Do mesmo modo, o estágio médio registrou maior número de espécies secundárias iniciais e clímax, evidenciando variações na composição de sua sinúsia (Leitão-Filho 1993). Já o estágio avançado, apresentou maior riqueza de espécies secundárias tardias e indivíduos clímax, corroborando estudos realizados por Gandolfi (1991) e Leitão Filho (1993). 
Nos três estágios estudados, as classes de diâmetro entre 5-7,9 cm foram as mais abundantes. Esse resultado vem ao encontro de trabalhos realizados em florestas, onde há maior número de indivíduos concentrados nas menores classes de diâmetro, representada em gráfico na curva J- invertido (Budke et al. 2004; Colonetti et al. 2009; Marchi e Jarenkow 2008). Embora tenha maior número de indivíduos nas menores classes de diâmetro, foram amostrados espécimes com DAP de $70 \mathrm{~cm}$. Estudos regionais também tiveram resultados semelhantes para os diâmetros de espécies florestais (Colonetti et al. 2009; Bosa et al. 2015). A altura média, registrada nos três estágios, ficou entre 6-7,9 m. Muitos trabalhos, em condições semelhantes, observaram maior distribuição de altura entre 5 e $10 \mathrm{~m}$, ratificando os dados obtidos neste estudo (Bosa et al. 2015; Colonetti et al. 2009; Marchi e Jarenkow 2008).

Neste estudo, não foi possível observar a relação das espécies ameaçadas com o estágio sucessional. No entanto, a redução na extensão fitogeográfica e o elevado grau de degradação de grande parte dos remanescentes de floresta podem contribuir para uma redução no tamanho populacional das espécies e se tratando de espécies ameaçadas a problemática pode acarretar em extinção local.

\section{Considerações FINAIS}

Com os dados obtidos neste estudo, fica evidente que os fragmentos florestais ao longo da bacia hidrográfica do rio Urussanga se encontram em pleno processo de restauração ambiental, necessitando de mais estudos, a fim de avaliar a continuidade dos serviços ecossistêmicos desempenhados pela vegetação ciliar.

\section{Agradecimentos}

À Coordenação de Aperfeiçoamento de Pessoal de Nível Superior (CAPES) pelas bolsas de estudos concedidas aos três primeiros autores. À Universidade do Extremo Sul Catarinense pela infraestrutura oferecida para realização deste trabalho.

\section{REFERÊNCIA}

Alvares CA, Stape JL, Sentelhas PC, de Moraes Gonçalves, JL, Sparovek G. 2013. Köppen's climate classification map for Brazil. Meteorologische Zeitschrift 22 (6): 711-728. https://doi.org/10.1127/0941-2948/2013/0507

Back Á.J. 2009. Caracterização Climática, In: Mineração de Carvão, Meio Ambiente E Desenvolvimento Sustentável No Sul de Santa Catarina: Uma Abordagem Interdisciplinar. pp. 17-33.

Bosa DM, Pacheco D, Pasetto MR, Santos R. 2015. Florística e estrutura do componente arbóreo de uma floresta ombrófila densa montana em Santa Catarina, Brasil. Revista Árvore, 39 (1): 49-58. https://doi.org/10.1590/0100-67622015000100005

Brackmann CE, Freitas EM. 2013. Florística arbórea e arbustiva de um fragmento de Mata Ciliar do arroio Boa Vista, Teutônia, RS, Brasil. Hoehnea 40 (2): 365-372. https://doi.org/10.1590/S2236-89062013000200007

Budke JC, Giehl ELH, Athayde, EA, Eisinger SM, Záchia RA. 2004. Florística e fitossociologia do componente arbóreo de uma floresta ribeirinha, arroio Passo das Tropas, Santa Maria, RS, Brasil. Acta Botânica Brasilica, 18 (2): 581-589. https:// doi.org/10.1590/S0102-33062004000300016 
Budowski G. 1965. Distribution of tropical American rain forest species in the light of successional processes. Turrialba, $15,40-42$.

Citadini-Zanette V, Santos R, Emerich KH, Pasetto MR, Cemin JG, Fernandes, MB. 2014. Composição florística de um fragmento florestal ciliar no Sul de Santa Catarina. Revista Tecnologia e Ambiente, 20, 55-70. https://doi.org/10.18616/ ta.v20i0.1560

Citadini-Zanette V, Santos R, Emerich KH, Pasetto MR, Cemin JG, Fernandes MB. 2014.

CNCFLORA. Lista Vermelha da flora brasileira. Centro Nacional de Conservação da Flora. Disponível em: Acesso em: $10 \mathrm{dez} .2019$

Composição florística de um Fragmento florestal Ciliar no Sul de Santa Catarina. Revista Tecnologia e Ambiente, 20, 5570. https://doi.org/10.18616/ta.v20i0.1560

Colonetti S, Citadini-Zanette V, Martins R, Santos, Rocha E, Jarenkow JA. 2009. Florística e estrutura fitossociológica em floresta ombrófila densa submontana na barragem do rio São Bento, Siderópolis, Estado de Santa Catarina. Acta Scientiarum, 31 (4): 397-405. https://doi.org/10.4025/actascibiolsci.v31i4.3345

Connell JH., 1978. Diversity in Tropical Rain Forests and Coral Reefs. Science, 199 (4335): 1302-1310. https://doi. org/10.2307/1745369

Elias GA, Corrêa P, Citadini-Zanette V, Santos R. 2015. Arecaceae: Análise bibliométrica das espécies nativas do estado de Santa Catarina. Ciência e Natura, 37 (1): 85-92. https://doi.org/10.5902/2179460X15399

Elias GA, Santos. 2016. Produtos florestais não madeireiros e valor potencial de exploração sustentável da Floresta Atlântica no sul de Santa Catarina. Ciência Florestal, 26 (1): 249-262. https://doi.org/10.5902/1980509821117

Fernández D, Barquín J, Álvarez-Cabria M, Peñas FJ. 2014. Land-use coverage as an indicator of riparian quality. Ecological Indicators, 41, 165-174. https://doi.org/10.1016/J.ECOLIND.2014.02.008

Fierke MK, Kauffman JB. 2005. Structural dynamics of riparian forests along a black cottonwood successional gradient. Forest Ecology and Management, 215 (1-3): 149-162. https://doi.org/10.1016/J.FORECO.2005.06.014

Jia X, Wang Y, Shao M, Luo Y, Zhang C, 2017. Estimating regional losses of soil water due to the conversion of agricultural land to forest in China's Loess Plateau. Ecohydrology, 10 (6): 18-51. https://doi.org/10.1002/eco.1851

Klein AS, Citadini-Zanette V, Lopes RP, Santos. 2009. Regeneração natural em área degradada pela mineração de carvão em Santa Catarina, Brasil. Revista Escola de Minas, 62 (3): 297-304. https://doi.org/10.1590/S0370-44672009000300007

Leitão Filho H, Pagano SN. 1993. Ecologia da Mata Atlântica em Cubatão, SP. Editora UNESP, Fundação para o Desenvolvimento da UNESP, $184 \mathrm{p}$.

Liu Y, Miao HT, Huang Z, Cui Z, He H, Zheng J, Han F, Chang X, Wu GL. 2018. Soil water depletion patterns of artificial forest species and ages on the Loess Plateau (China). Forest Ecology and Management, 417, 137-143. https://doi. org/10.1016/J.FORECO.2018.03.005 
Lopes WP, Silva AF, Souza AL, Meira Neto JAA. 2002. Estrutura fitossocióloga de um trecho de vegetação arbórea no Parque Estadual do Rio Doce - Minas Gerais , Brasil. Acta Botânica Brasilica, 16 (4): 443-456. https://doi.org/10.1590/ S0102-33062002000400007

Marchi TC, Jarenkow JA. 2008. Estrutura do componente arbóreo de mata ribeirinha no rio Camaquã, município de Cristal, Rio Grande do Sul, Brasil. Iheringia. Série Botânica, 63 (2): 241-248.

Molino JF, Sabatier D. 2001. Tree Diversity in Tropical Rain Forests: A Validation of the Intermediate Disturbance Hypothesis. Science, 23; 294 (5547): 1702-1704. https://doi.org/10.1126/science.1060284

Moro MF; Martins FR. 2011. Métodos de levantamento do componente arbóreo-arbustivo. In: Felfili JM; Eisenlohr PV; Melo MM da RF; de Andrade LA; de Meira Neto JAA. (Eds.), Fitossociologia no Brasil: Métodos e Estudos de Caso. Viçosa, Editora da Universidade Federal de Viçosa.

Mueller-Dombois D, Ellenberg H. 2002. Aims and methods of vegetation ecology, 2 ed. ed. Wiley.196p.

Oliveira-Filho AT. 2009. Classificação das fitofisionomias da América do Sul cisandina tropical e subtropical: proposta de um novo sistema - prático e flexível - ou uma injeção a mais de caos? Rodriguésia, 60 (2) 237-258. https:/ /doi.org/10.1590/21757860200960201

Orlowski N, Kraft P, Pferdmenges J, Breuer L. 2016. Exploring water cycle dynamics by sampling multiple stable water isotope pools in a developed landscape in Germany. Hydrology and Earth System Sciences, 20 (9): 3873-3894. https:// doi.org/10.5194/hess-20-3873-2016

Peña-Claros M. 2003. Changes in Forest Structure and Species Composition during Secondary Forest Succession in the Bolivian Amazon. Biotropica, 35 (4): 450. https://doi.org/10.1646/01078

Ravazzoli C. 2013. A Problemática Ambiental do Carvão em Santa Catarina: Sua Evolução Até os Termos de Ajustamento de Conduta Vigente Entre os Anos de 2005 e 2010. Geografia em Questão, 6 (1): 179-201.

Reitz R, Klein RM. 1974. Arecaceae, in: Reitz, R. (Ed.), Flora Ilustrada Catarinense. Herbário Barbosa Rodrigues, Itajaí, pp. $1-189$.

Reitz R, Klein RM. 1964. O reino vegetal de Rio do Sul. Sellowia, 16, 9-118.

Ribeiro TM, Martins SV, Lana VM, Silva KA. 2011. Sobrevivência e crescimento inicial de plântulas de Euterpe edulis Mart. transplantadas para clareiras e sub-bosque em uma Floresta Estacional Semidecidual, em Viçosa, MG. Revista Árvore, 35 (6): 1219-1226. https://doi.org/10.1590/S0100-67622011000700008

Rodrigues RR, Shepherd GJ. 1992. Análise da variação estrutural e fisionômica da vegetação e das características edáficas, num gradiente altitudinal na Serra do Japi: Jundiaí (SP), in: História Natural Da Serra Do Japi: Ecologia E Preservação de Uma Área Florestal No Sudeste Do Brasil. pp. 64-97.

Rodrigues VHP, Lopes S, Araújo GM, Schiavini I. 2010. Composição, estrutura e aspectos ecológicos da floresta ciliar do rio Araguari no Triângulo Mineiro. Hoehnea, 37 (1): 87-105. https://doi.org/10.1590/S2236-89062010000100006

Santos OA, Couceiro SRM, Rezende ACC, Silva. 2016. Composition and richness of woody species in riparian forests in urban areas of Manaus, Amazonas, Brazil. Landscape and Urban Planning, 150, 70-78. https://doi.org/10.1016/J. 
Santos R., Citadini-Zanette V, Leal-Filho LS, Hennies WT. 2008. Spontaneous Vegetation on Overburden Piles in the Coal Basin of Santa Catarina, Brazil. Restoration Ecology, 16 (3): 444-452. https://doi.org/10.1111/j.1526-100X.2007.00309.x

Sawczuk AR, Figueiredo Filho A, Dias AN, Watzlawick LF, Stepka TF. 2012. Alterações na estrutura e na diversidade florística no período 2002-2008 de uma floresta ombrófila mista montana do centro-sul do Paraná, Brasil. Floresta, 42 (1): 1-10. https://doi.org/10.5380/rf.v42i1.26286

Sevegnani L, Silva TC, Gasper AL, Meyer L, Verdi M. 2012. Flora Arbórea e o Impacto Humano nos Fragmentos Florestais na Bacia do Rio Pelotas, Santa Catarina, Brasil. Rev. Estud. Ambient. 14 (1): 60-73. https://doi.org/10.7867/19831501.2012V14N1P60-73

Sijtsma K, Emons WHM. 2010. Nonparametric statistical methods, in: International Encyclopedia of Education, Wiley Series in Probability and Statistics. Wiley, pp. 347-353. https://doi.org/10.1016/B978-0-08-044894-7.01353-1

Smith L, Downs R, Klein RM. 1988. Euforbiáceas, in: Reitz, R. (Ed.), Flora Ilustrada Catarinense. pp. 1-408.

Sun J, Yu X, Wang H, Jia G, Zhao Y, Tu Z, Deng W, Jia J, Chen J. 2018. Effects of forest structure on hydrological processes in China. Journal of Hydrology, 561, 187-199. https://doi.org/10.1016/j.jhydrol.2018.04.003

Trindade MJ, Lameira OA. 2015. Espécies úteis da família Euphorbiaceae no Brasil. Revista Cubana de Plantas Medicinais, $19,1-23$.

Venzke TS, Martins SV. 2013. Aspectos florísticos de três estágios sucessionais em mata ciliar em Arroio do Padre, extremo sul do Brasil. Floresta 43, 191-204. https://doi.org/10.5380/rf.v43i2.27667 